\section{Analysis of Efficiency of Overhead Irrigation in Container Production}

\author{
R.C. Beeson, Jr., and G.W. Knox \\ University of Florida, Central Florida Research and Education Center- \\ Sanford, 2700 East Celery Avenue, Sanford, FL 32771
}

Additional index words. Rhododendron sp., Pittosporum tobira, impact sprinkler, wobbler sprinkler, azalea, pittosporum, container spacing

\begin{abstract}
Volume of water captured in a container as a function of sprinkler type, spacing, plant type, and container size was measured for marketable-sized plants. Percent water captured was calculated and a model to predict this value derived. Percent water captured was inversely related to the leaf area contained in the cylinder over the container when containers were separated, and with total plant leaf area at a pot-to-pot spacing. This relationship was independent of leaf curvature (concave vs. convex). Canopy densities were less related to percent water captured than leaf areas. Irrigation application efficiencies separated by spacing ranged from $37 \%$ at a close spacing to $25 \%$ at a spacing of $7.6 \mathrm{~cm}$ between containers. Container spacing, canopy shedding, and possibly some canopy retention of water later lost by evaporation were determined to be the main factors associated with the low efficiencies. The results suggest that higher irrigation application efficiencies would be maintained only if plants were transplanted to larger containers before reaching maximum canopy size rather than spacing existing containers to achieve more room for canopy growth.
\end{abstract}

Overhead irrigation is the most practical and the most commonly used irrigation system for container production of woody ornamentals. In practice, many nursery growers use drip or microjet irrigation for material grown in containers $>20$ liter. Below this size, labor and material cost for the installation and maintenance of an individual-type irrigation system are considered prohibitive.

In Florida, the availability of water is a serious problem that is predicted to become critical around the turn of the century. With an average rainfall of $\approx 1000$ to 1250 $\mathrm{mm} \cdot \mathrm{year}^{-1}$, container nurseries apply an additional 1420 to $3040 \mathrm{~mm}$ of water annually to produce marketable plants (Harrison, 1976). The irrigation application efficiency (amount of water retained in the container and available to the plant/total water applied) of overhead irrigation is, at best, near $80 \%$ and drops rapidly with increased spacing between pots (Furuta, 1976). Weatherspoon and Harrell (1980) reported that irrigation application efficiencies of impact sprinklers averaged $26 \%$ over the course of a year, and were reduced to $13 \%$ using a spinner-type sprinkler. Our objective was to determine the factors that result in these low application efficiencies for overhead irrigation. The results are discussed on the basis of sprinkler type as a function of container spacing, pot size, and canopy characteristics.

Three landscape plant types were used in this experiment: Rhododendron spp. 'Duc du

Received for publication 2 July 1990. Florida Agricultural Experiment Stations Journal Series no. R-00900. Special appreciation is given to Jon's Nursery for the use of their facilities and plant material. The cost of publishing this paper was defrayed in part by the payment of page charges. Under postal regulations, this paper therefore must be hereby marked advertisement solely to indicate this fact. weighed before being placed under the plant.

The experiment was performed in a section of an existing commercial nursery. Irrigation lines were $7.7 \mathrm{~m}$ apart with risers extending $1.23 \mathrm{~m}$ above the ground in a staggered pattern. To insure consistent coverage, four lines covering $710 \mathrm{~m}^{2}$ were used. Before placing the plants, a coefficient of uniformity was determined for both impact (Model 3023-1-3/4" M, \#8 nozzle, 3.18$\mathrm{mm}$ orifice, Senninger Irrigation, Orlando, Fla.) and off-center rotary-action sprinklers (Wobbler, \#8 nozzle, 3.18-mm orifice, Senninger Irrigation) by changing the sprinkler heads on existing risers. Coefficients of uniformity were 88.1 for the impact sprinklers and 92.3 for the wobbler sprinklers, determined over the experimental $280-\mathrm{m}^{2}$ area at a spacing of $1.54 \mathrm{~m}$ between sampling cups. This corresponds to concurrent determinations of 87.3 and 93.0 for impact and wobbler sprinklers, respectively, by Senninger Irrigation engineers using a $0.67-\mathrm{m}$ cup spacing over a $63-\mathrm{m}^{2}$ subsection between sprinklers (A. Skolnik, personal communication).

Based on the map of the irrigation pattern derived from the 1.54-m spacing of the sampling cups, nine areas were selected that provided the most uniform water distribution for both sprinkler types. Ten catch plants of each species $\times$ size category were randomly arranged in the interior of each area and surrounded by similar plants to fill out the area. Containers were placed pot-to-pot at right angles to each other. Sampling cups were placed around three sides of each area to measure the actual amount of water each area received. The irrigation system was operated for $\approx 1 \mathrm{~h}$ at $17.0 \mathrm{kPa}$, then stopped. The plants were allowed 15 min to drip dry, and the sponges were removed and placed in ziplock bags (Dow Chemical, Indianapolis, Ind.). In those plants where the water captured on the plastic sheet was greater than sponge capacity, water was expressed from the sponge by hand into the bag, and the sponge used to absorb the excess water. The sponges were sealed in the bags with the captured water.

The first irrigation set consisted of impact sprinklers with the containers placed pot-topot in an area $\approx 4 \mathrm{~m}^{2}$ (close spacing). This was then repeated with wobbler sprinklers.

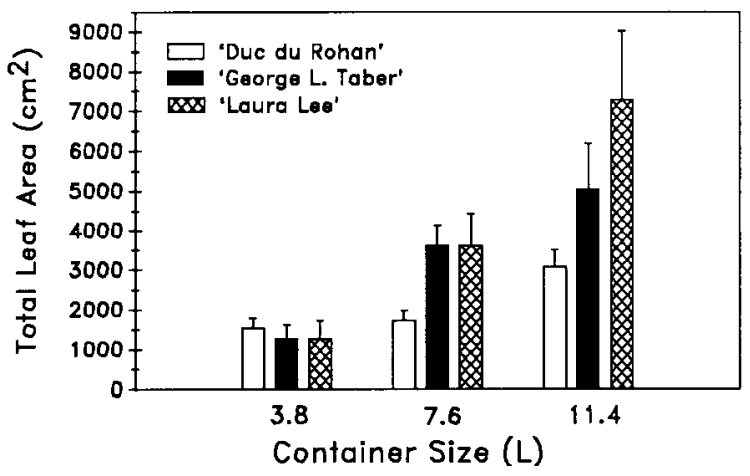

Fig. 1. Mean total leaf areas of the capture plants as a function of plant type and container size. Each mean represents 10 repetitions. 

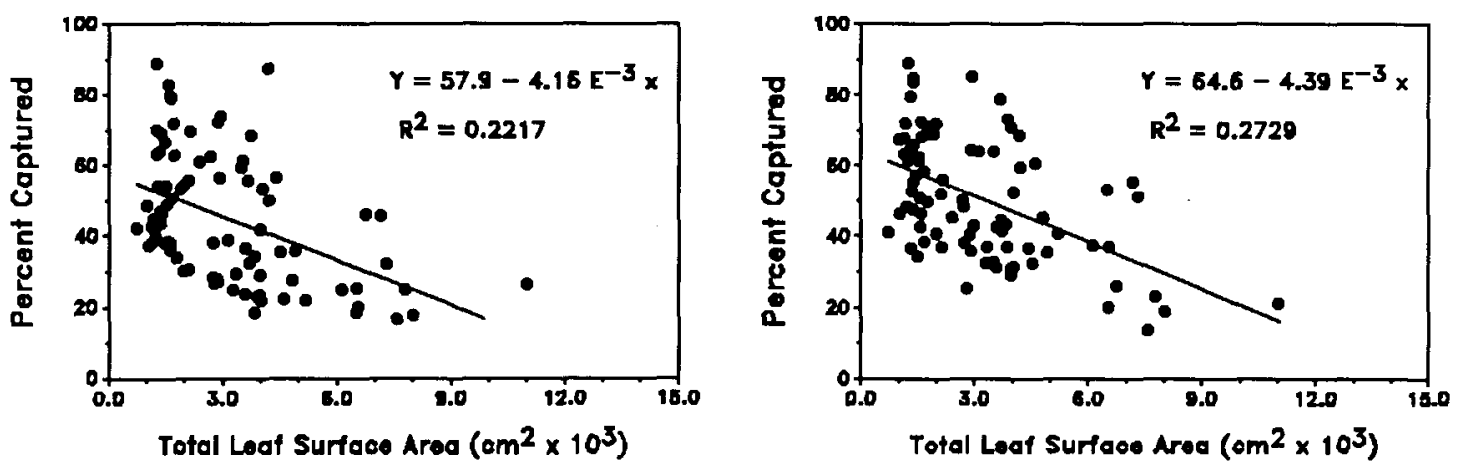

C

D
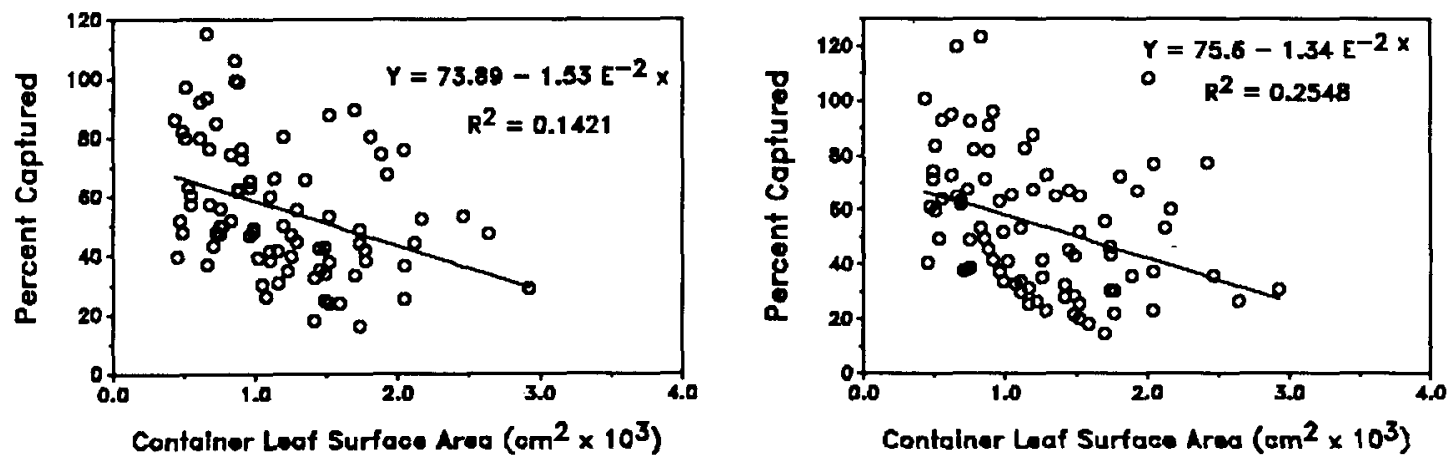

Fig. 2. Scatter plots of percent irrigation water captured as a function of total leaf surface for close spacing (A,B) and container leaf surface area for separate spacing $(\mathbf{C , D})$. The data were separated according to the type of sprinkler-wobblers $(\mathbf{A}, \mathbf{C})$ or impact $(\mathbf{B}, \mathbf{D})$. The linear regression equation and corresponding regression coefficient for each data set are given. All lines were significant at $P=0.01$.

Afterward, the containers were spaced 7.5 $\mathrm{cm}$ apart at right angles in an area of $5 \mathrm{~m}^{2}$ (separated spacing). Excess plants were removed. The plants were irrigated again and water captured as before. Impact sprinklers were reinstalled and the irrigation repeated. About $2 \mathrm{~h}$ was required to collect the water and rearrange the plants after each irrigation; therefore, canopies were dry at the beginning of each irrigation.

For each catch plant, the horizontal top canopy surface was estimated by measuring the widest diameter and the diameter at right angles to this. These were then averaged and top canopy surface was calculated as a circle using the average. The leaves from each plant were removed and the leaf area determined using a LI-COR leaf area meter (LI-COR, Lincoln, Neb.). Leaves were separated into leaf area inside the cylinder of the container (container leaf area; $\mathrm{cm}^{2}$ ) and the total leaf area of the canopy $\left(\mathrm{cm}^{2}\right)$. From these, plant leaf area indexes were computed for each plant over the pot (PLAI = container leaf area/surface area of the container), for the total canopy (TLAI = total leaf area/top canopy surface area), and for total canopy based on container surface area (Compress LAI = total leaf area/surface area of the container). The volume of water for each catch plant was calculated as: (weight of bag + sponge after irrigation) - (weight of bag + oven-dry sponge). The percentage water captured (\% captured) for each plant was calculated as: (volume of water captured per $100 \mathrm{~cm}^{2}$ of container medium surface area)/ (average volume of water captured in the sampling cups per $100 \mathrm{~cm}^{2}$ maximum surface area) for each sprinkler $\times$ container spacing irrigation.

The results were analyzed as a $2 \times 2 \times$ $3 \times 3$ factorial consisting of sprinkler type, spacing, species, and container size, respectively. Regression and multiple regression analysis were used to develop a model to predict percentage of water captured.

Significant differences were found within each sprinkler-spacing combination (Table 1). When the containers were separated, there was no significant difference in the \% captured between impact and wobbler sprinklers. However, with a close spacing, the use of wobbler sprinklers significantly improved $\%$ captured over that achieved using impact sprinklers, While the mean \% captured for impact sprinklers was higher for spaced plants, wobbler sprinklers gave the most consistent \% captured independent of container spacing. For the marketable-sized plants used here, close spacing (pot-to-pot) resulted in significantly less \% captured (47.6\%) than separated spacing $(56.3 \%)$. The close spacing compacted the plant canopies, thereby increasing the ability of a canopy to hold or deflect droplets. Even with a pot-topot spacing, containers covered only $78 \%$ of the bed surface area. Differences between the two sprinkler types may have resulted from differences in droplet size, trajectory angles, and droplet velocity. These may change at different system pressures.

The interaction between species and con- tainer size was also significant (Table 2). Percent captured ranged from $70.2 \%$ for 'Laura Lee' pittosporum in 3.8-liter containers to $30.3 \%$ for 'Laura Lee' in 11.4-liter containers. In general, the 3.8- and 7.6-liter containers had higher \% captured than the 11.4-liter. This difference was due to proportionally larger canopies in the largest containers (Fig, 1).

To account for differences in \% captured, linear regressions of \% captured were calculated using the plant canopy dimensions of: container leaf area, total leaf area, container surface area, top canopy surface area, PLAI, TLAI, and Compress LAI. These were grouped according to container size, spacing $\times$ container size, plant type, plant type $\times$ spacing, spacing, sprinkler type $\times$ spacing, and sprinkler type. When separated by container size, TLAI was the best estimator of $\%$ captured, being significantly correlated for all three sizes. However, when grouped by spacing $\times$ container size, PLAI was better correlated, though significant only for the pot-to-pot spacing. Grouping by plant type found total leaf area correlated with \% captured for 'Duc du Rohan' azalea and 'Laura Lee' but not 'George L. Taber' azalea. When subgrouped by spacing (plant type $\times$ spacing), significant correlations were found between \% captured and total leaf area for both Rhododendron spp. placed pot-to-pot, but only for 'George L. Taber' and 'Laura Lee' at a separated spacing. Separated by spacing, total leaf area showed the best relationship to $\%$ captured with the close spacing. At the 
Table 1. Percent water captured in containers averaged over plant types and container sizes, but separated by sprinkler type and container spacing.

\begin{tabular}{lcc}
\hline \hline & & Spacing $^{z}$ \\
\cline { 2 - 3 } Sprinkler type & Close $^{\mathbf{y}}$ & Separated $^{\mathbf{x}}$ \\
\hline Impact & $44.3 \pm 17.8$ & $58.0 \pm 26.3$ \\
Wobbler & $51.1 \pm 16.9$ & $54.6 \pm 24.3$ \\
Contrast & & \\
Spacing & Close & Separated \\
Wobbler vs. impact & $* *$ & NS \\
Sprinkler type & Impact & Wobbler \\
Close vs. separated & $*$ & NS \\
\hline
\end{tabular}

${ }^{2}$ Means and standard errors $(\mathrm{n}=90)$.

'Pot-to-pot spacing at $90^{\circ}$ to each other.

${ }^{*}$ Containers spaced $7.6 \mathrm{~cm}$ apart.

"Single-degree-of-freedom contrast.

Nonsignificant or significant at $P=0.05$ or 0.01 , respectively.

Table 2. Percent water captured in containers separated by plant type and container size and averaged over spacing and sprinkler type.

\begin{tabular}{|c|c|c|c|}
\hline \multirow[b]{2}{*}{ Plant type } & \multicolumn{3}{|c|}{ Container size (liter) ${ }^{z}$} \\
\hline & 3.8 & 7.6 & 11.4 \\
\hline \multicolumn{4}{|l|}{ Azalea } \\
\hline Duc du Rohan & $62.3 \pm 17.5$ & $59.1 \pm 18.5$ & $42.2 \pm 17.9$ \\
\hline George L. Taber & $57.3 \pm 19.6$ & $62.9 \pm 24.4$ & $47.0 \pm 23.9$ \\
\hline \multicolumn{4}{|l|}{ Pittosporum } \\
\hline Laura Lee & $70.2 \pm 17.0$ & $36.8 \pm 11.1$ & $30.3 \pm 14.8$ \\
\hline \multicolumn{4}{|l|}{ Contrast $^{y}$} \\
\hline Rohan vs. Taber & NS & NS & NS \\
\hline Rohan vs. L. Lee & NS & $* *$ & $* *$ \\
\hline Taber vs. L. Lee & $* *$ & $* *$ & $* *$ \\
\hline Container size & Duc du Rohan & G. Taber & Laura Lee \\
\hline 3.8 vs. 7.6 & $\overline{\text { NS }}$ & NS & $* *$ \\
\hline 3.8 vs. 11.4 & $* *$ & $*$ & $* *$ \\
\hline 7.6 vs. 11.4 & $* *$ & $* *$ & NS \\
\hline
\end{tabular}

${ }^{2}$ Means and standard errors $(n=40)$.

${ }_{\mathrm{NS} \text { **** }}^{\mathrm{S}}$ Sing-degree-of-freedom contrast.

Nonsignificant or significant at $P=0.05$ or 0.01 , respectively.

Table 3. Effect of container size and spacing between containers on the percentage of bed surface area covered by circular containers. Calculations were based on the percent surface area of each container size placed within a square area, assuming linear alignment. The sides of the square were equal to space between containers + container diameter.

\begin{tabular}{lccr}
\hline \hline \multirow{2}{*}{$\begin{array}{l}\text { Space between } \\
\text { containers }(\mathrm{cm})\end{array}$} & 3.8 & 7.6 & Container size (liter) \\
\cline { 2 - 4 } 0.0 & 78.5 & 78.5 & $\mathbf{1 1 . 4}$ \\
2.5 & 59.4 & 63.6 & 78.5 \\
5.1 & 46.4 & 52.5 & 66.1 \\
7.6 & 37.3 & 44.1 & 56.4 \\
10.2 & 30.6 & 37.6 & 48.7 \\
12.7 & 25.6 & 32.3 & 42.5 \\
Regression & & & 37.4 \\
3.8 liter & $\%$ bed area $=0.72$ & 0.103 container separation (cm) \\
7.6 liter & $\%$ bed area $=0.72-0.091$ container separation (cm) \\
11.4 liter & $\%$ bed area $=0.72-0.081$ container separation (cm) & \\
\hline
\end{tabular}

${ }^{2}$ Regression equations to calculate percentage of bed area covered by container as determined by data presented above.

separated spacing, container leaf area was more highly related. This relationship is reasonable considering the size of the plants. In the close spacing, plant canopies were compressed. Therefore, more leaf area existed over the container than would have been measured. When grouped by sprinkler type and spacing, the relationship to spacing did not change.

Multivariate regression equations were calculated using the previously noted plant dimensions and container size. Container size was chosen because of its independence of the plant characteristics and was not used to subgroup the data. The addition of container size to the models did not significantly improve the relationship between $\%$ captured and total leaf area or container leaf area. The relationships of TLAI and PLAI to \% captured were significantly improved, but still had lower $r^{2}$ values than the simple relationship of total leaf area or container leaf area.

The most satisfactory predictive models were obtained when the data were separated by sprinkler type and spacing (Fig. 2). Regression intercepts were significantly different, but the slopes were not. While all relationships were significant $(>95 \%)$, the relationship of container leaf area to \% captured was the weakest for wobbler sprinklers with separated spacing (Fig. 2C). None of the canopy characteristics calculated were well correlated with $\%$ captured for wobblers with a separated spacing. This result may have been due to leaks occurring in the polyethylene sheet that sealed the pot surface. However, this appears unlikely since this was the third irrigation set and good correlations were found for the fourth set. Because this irrigation occurred in midafternoon, light winds or elevated temperatures may have weakened the canopy effect. Averaged over the entire data set, the model for predicting \% captured was calculated to be: \% captured $=67.73-9.9 \times 10^{-3} \mathrm{c} \mathrm{m}^{2}$ pot $\left(R^{2}=\right.$ $0.1781, P>0.95)$. In the above, $\%$ captured represents the percentage of water reaching the container medium surface relative to the maximum amount of water that would fall into an isolated empty container. As the containers are spaced farther apart, the percentage of bed surface covered by the containers diminishes rapidly (Table 3 ). Thus, to determine the actual irrigation application efficiency at a given spacing, the \% captured above must be multiplied by the percentage of bed surface area covered by the containers. For example, the \% captured for 'George L. Taber' azaleas grown in 7.6-liter containers was $62.9 \%$. However, the actual irrigation application efficiency given a 7.6$\mathrm{cm}$ spacing, was only $27.7 \%[(0.629 \times$ $0.441) \times 100]$. The same spacing for the 11.4-liter 'Laura Lee' calculates to only a $15 \%$ irrigation application efficiency. These values agree broadly with the $26 \%$ average reported by Weatherspoon and Harrell (1980).

From these experiments, we conclude that water shedding and possibly water holding by the canopy and the spacing of the containers are the principal causes of low irrigation application efficiencies in container nurseries. Leaf curvature (concave vs. convex) differed between the two azalea cultivars but was not a major factor in \% captured. These results suggest that overhead irrigation application efficiency is adequate when containers are placed pot-to-pot and the plant material is small. However, as the plants grow, increasing leaf area decreases the percentage of water reaching the container. Once plants have reached a marketable size, even with pot-to-pot spacing, the \% captured decreases to an average of $37 \%$. Spacing the containers at this stage improves the mean $\%$ captured, but the irrigation application efficiency is further reduced. These results suggest that irrigation application efficiency could be maintained if plants were transplanted to larger containers maintained at a pot-to-pot spacing for as long as growth permits.

\section{Literature Cited}

Furuta, T. 1976. Environmental plant production and marketing. Cox Publishing, Arcadia, Calif. p. 94-156.

Harrison, D.S. 1976. Irrigation water applied to three commercial ornamental container nurseries. Proc. Fla. State Hort. Soc. 90:306-308.

Weatherspoon, D.M. and C.C. Harrell 1980. Evaluation of drip irrigation for container production of woody landscape plants. HortScience 15:488-489. 